\title{
Producción de genotipos de frijol negro en condiciones de humedad residual y sequía terminal
}

\section{Production of black bean genotypes under residual moisture and terminal drought conditions}

\author{
Oscar Hugo Tosquy Valle ${ }^{1}$, Ernesto López Salinas ${ }^{1}$, Rigoberto Zetina Lezama ${ }^{1 \ddagger}$, \\ Bernardo Villar Sánchez ${ }^{2}$ y José Raúl Rodríguez Rodríguez ${ }^{3}$
}

\footnotetext{
${ }^{1}$ Campo Experimental Cotaxtla. CIRGOC, INIFAP. Km 34.5 Carretera Federal Veracruz-Córdoba. 94270 Medellín de Bravo, Veracruz, México.

*Autor responsable (zetina.rigoberto@inifap.gob.mx)

${ }^{2}$ Campo Experimental Centro de Chiapas, CIRPAS, INIFAP. Km 3 Carretera Ocozocoautla-Cintalapa. 29140 Ocozocoautla de Espinosa, Chiapas, México.

${ }^{3}$ Campo Experimental Ixtacuaco. CIRGOC, INIFAP. Km 34.5 Carretera Federal Martínez de la Torre-Tlapacoyan, Col. Rojo Gómez. 93600 Martínez de la

Torre, Veracruz, México.
}

\section{RESUMEN}

En Veracruz y Chiapas, el cultivo de frijol del ciclo otoño-invierno, sufre periodos de sequía durante la etapa reproductiva, lo que causa bajos rendimientos. El objetivo de esta investigación fue determinar el rendimiento de genotipos de frijol, evaluados bajo condiciones de humedad residual y de sequía terminal durante el ciclo otoño-invierno de 20132014, en las localidades de La Candelaria, Medellín, Veracruz, Campo Experimental Ixtacuaco (CEIXTA), municipio de Martínez de la Torre, Veracruz, y Campo Experimental Centro de Chiapas (CECECH), municipio de Ocozocoautla, Chiapas. Se evaluaron 11 líneas avanzadas de frijol, comparadas con las variedades Negro Tacaná y Negro Jamapa, en diseño experimental bloques al azar con tres repeticiones. Las variables de respuesta fueron: días a floración media, días a madurez fisiológica, peso de 100 semillas y rendimiento de grano. En la Candelaria y el CECECH, ambas con ocurrencia de sequía terminal, se obtuvieron valores significativamente inferiores en las cuatro variables de respuesta, con respecto a los obtenidos en el CEIXTA, sin estrés por sequía. Las líneas SEN-56, SCN-2, SEN-70 y NCB-229 fueron las más precoces en alcanzar su floración media en las tres localidades de prueba, la cual en promedio ocurrió antes de 35 días después de la siembra. Las tres primeras líneas también mostraron menor tiempo a la madurez fisiológica, en promedio antes de los 71 días. La línea SCN-2 obtuvo en promedio el mayor peso de 100 semillas ( $27 \mathrm{~g}$ ), el cual fue significativamente superior a los obtenidos por el resto de los genotipos, que registraron pesos menores a 25 g. Así mismo SEN-70, NGO-17-99 y Jamapa Plus,

Cita recomendada:

Tosquy Valle, O. H., E. López Salinas, R. Zetina Lezama, B. Villar Sánchez y J. R. Rodríguez Rodríguez. 2017. Producción de genotipos de frijol negro en condiciones de humedad residual y sequía terminal. Terra Latinoamericana 35: 29-39. junto con otras cuatro líneas obtuvieron un rendimiento promedio mayor a $1000 \mathrm{~kg} \mathrm{ha}^{-1}$, significativamente superior al de las variedades Negro Jamapa y Negro Tacaná. SEN-70 fue la de mayor rendimiento con y sin sequía terminal.

Palabras clave: mejoramiento genético, rendimiento, Phaseolus vulgaris.

\section{SUMMARY}

In Veracruz and Chiapas, autumn-winter growing cycle bean crops suffer periods of drought during the reproductive stage, causing low yields. The aim of this study was to determine the yield of bean genotypes evaluated under conditions of residual moisture and terminal drought during the autumn-winter 20132014 growing cycle, in the localities of La Candelaria, Medellín, Veracruz; Ixtacuaco Experiment Station (CEIXTA), municipality of Martínez de la Torre, Veracruz; and Centro de Chiapas Experiment Station (CECECH), municipality of Ocozocoautla, Chiapas. Eleven advanced bean lines were evaluated and compared with the control varieties Negro Tacaná and Negro Jamapa in a randomized block experimental design with three replications. The response variables were days to average flowering, days to physiological maturity, weight of 100 seeds and grain yield. In La Candelaria and the CECECH, both with terminal drought occurrence, the four response variables were significantly lower than those obtained in the CEIXTA, without drought stress. The SEN-56, SCN-2, SEN-70 and NCB-229 lines had significantly earlier average flowering in the three test locations, which on average

Recibido: enero de 2016. Aceptado: octubre de 2016. Publicado en Terra Latinoamericana 35: 29-39. 
occurred before 35 days after sowing. The first three lines also showed shorter time to physiological maturity, which on average was less than 71 days. The SCN-2 line produced the highest average weight of 100 seeds $(27 \mathrm{~g})$, which was significantly higher than those obtained with the other genotypes, which had weights below $25 \mathrm{~g}$. In addition, SEN-70, NGO-17-99 and Jamapa Plus, along with four other lines obtained an average yield of more than $1000 \mathrm{~kg} \mathrm{ha}^{-1}$, which was significantly higher than those of the varieties Negro Jamapa and Negro Tacaná. SEN-70 was the highestyielding line with and without occurrence of terminal drought.

Index words: genetic improvement, yield, Phaseolus vulgaris.

\section{INTRODUCCIÓN}

El frijol común Phaseolus vulgaris L. constituye uno de los granos más importantes para la alimentación humana, a nivel mundial se siembran aproximadamente 26 millones de hectáreas (Emam et al., 2010). En México, durante el ciclo otoño-invierno 2012-13 con humedad residual, se sembraron 122840 ha, con un rendimiento promedio de $0.81 \mathrm{Mg} \mathrm{ha}^{-1}$ (SIAP, 2014). En el mismo ciclo agrícola, en los estados de Chiapas y Veracruz se sembraron 62055 ha de frijol, equivalente al $50.5 \%$ de la superficie sembrada con humedad residual en el país, aunque el rendimiento promedio fue bajo $\left(0.65 \mathrm{Mg} \mathrm{ha}^{-1}\right)$, inferior al promedio nacional (SIAP, 2014).

Los bajos rendimientos obtenidos durante el ciclo otoño-invierno, obedecen en gran medida, al estrés hídrico que sufren las plantas de frijol, provocado por la ocurrencia de sequía, denominada terminal, porque generalmente se presenta después de la floración del cultivo, cuando el frijol es más sensible a la escasez de humedad (Acosta et al., 1999; Frahm et al., 2003). La sequía durante la etapa de floración, provoca aborto de flores y en la etapa de formación de vainas, aborto y poco desarrollo de las mismas, así como reducción de fotosíntesis, que provoca aborto del embrión (Manjeru et al., 2007). La sequía reduce la producción de materia seca y los componentes de rendimiento, a través de la disminución del área foliar y los días de llenado del grano (Emam et al., 2010), se afecta la formación del grano y su tamaño (Acosta-Díaz et al., 2011) y se acelera la madurez fisiológica y la senescencia del cultivo, por lo que hay una reducción significativa del rendimiento de grano (Acosta-Díaz et al., 2004; Manjeru et al., 2007). También existe reducción en el número de vainas por planta, causada por la abscisión de flores y vainas pequeñas o por falla en la fertilización por polen inviable (Manjeru et al., 2007).

En trabajos experimentales realizados en el centro de Veracruz, con genotipos de frijol negro cultivados con suspensión de riego al inicio de la etapa reproductiva (sequía terminal), se tuvo una reducción de 10 días en su ciclo de madurez fisiológica, una disminución del $50 \%$ en el número de vainas producidas por planta $\mathrm{y}$ de $62 \%$ en el rendimiento de grano, con respecto a los que fueron cultivados con riego durante todo su ciclo de cultivo (López-Salinas et al., 2011). En siembras comerciales de frijol, las pérdidas de rendimiento son del 20 hasta el $100 \%$, de acuerdo con la duración y magnitud de la sequía (López-Salinas et al., 2008).

El mejoramiento genético para tolerancia o resistencia a sequía, es una alternativa viable y práctica para disminuir los efectos negativos de este factor ambiental (Frahm et al., 2003; Acosta-Díaz et al., 2011), mediante el desarrollo de germoplasma adaptado a condiciones de sequía (Acosta et al., 1999; Molina et al., 2001; Frahm et al., 2003; Acosta-Díaz et al., 2004; López-Salinas et al., 2011). Al respecto, Acosta-Díaz et al. (2004) mencionan que existe una variación genética entre los genotipos de frijol para las características fisiológicas y fenológicas, que confieren adaptación a las condiciones de sequía terminal. En el Programa de Frijol del Instituto Nacional de Investigaciones Forestales, Agrícolas y Pecuarias (INIFAP) en Veracruz, se dispone de líneas avanzadas y variedades mejoradas de frijol negro que han mostrado adaptación a las condiciones tropicales del sureste de México. El objetivo de este trabajo fue evaluar este grupo de genotipos, con la finalidad de determinar su rendimiento de grano en condiciones de humedad residual y de sequía terminal en el centro y norte de Veracruz y centro de Chiapas.

\section{MATERIALES Y MÉTODOS}

\section{Ambientes de Evaluación}

El estudio se realizó durante el ciclo otoñoinvierno 2013-2014, en tres localidades. La primera fue en el Ejido La Candelaria, municipio de Medellín de Bravo, ubicada en el centro de Veracruz, a $18^{\circ} 55^{\prime}$ 
$19.41 " \mathrm{~N}, 96^{\circ} 11$ '57.10" O, a una altitud de $22 \mathrm{~m}$. En esta localidad, el clima es cálido subhúmedo $\mathrm{Aw}_{0}(\mathrm{w})(\mathrm{g})$, con una precipitación pluvial anual de $1400 \mathrm{~mm}$ y temperatura media anual de $25.4{ }^{\circ} \mathrm{C}$ (García, 2004; Díaz-Padilla et al., 2006). La segunda el Campo Experimental Ixtacuaco (CEIXTA), ubicado en el municipio de Martínez de la Torre, en el norte de Veracruz, a $20^{\circ} 02^{\prime} 17.9^{\prime \prime} \mathrm{N}$ y $97^{\circ} 05^{\prime} 47.0^{\prime \prime} \mathrm{O}$, a una altitud de $92 \mathrm{~m}$. Su clima es cálido húmedo $\operatorname{Af}(\mathrm{m})$ (e), con una precipitación pluvial anual de $1743 \mathrm{~mm}$ y temperatura media anual de $24{ }^{\circ} \mathrm{C}$ (García, 2004; Díaz-Padilla et al., 2006). La tercera fue el Campo Experimental Centro de Chiapas (CECECH), municipio de Ocozocoautla de Espinosa, en el centro de Chiapas, a $16^{\circ} 46^{\prime} 50.3$ " N y $93^{\circ} 24^{\prime} 13.2$ " O, a una altitud de $805 \mathrm{~m}$. El clima es cálido húmedo $(\mathrm{A}) \mathrm{C}\left(\mathrm{w}_{1}\right)$ ig' con abundantes lluvias en verano, una precipitación pluvial anual entre 800 y $1000 \mathrm{~mm}$, con una media de $898 \mathrm{~mm}$, temperatura media anual de $23.6^{\circ} \mathrm{C}$ y media mensual superior a $18{ }^{\circ} \mathrm{C}$ (García, 2004; SerranoAltamirano et al., 2006).

De acuerdo a la clasificación agronómica de la Norma oficial Mexicana PROY-NOM-021RECNAT-2000 (Norma Oficial Mexicana, 2000), en el sitio de La Candelaria, en el estrato de 0 a $30 \mathrm{~cm}$ de profundidad, el suelo es de textura franca, con $\mathrm{pH}$ moderadamente ácido (5.99), alto en su contenido de materia orgánica $(2.44 \%)$, bajo en nitrógeno inorgánico $\mathrm{NH}_{4}^{+}+\mathrm{NO}_{3}^{-}\left(17 \mathrm{mg} \mathrm{kg}^{-1}\right)$, alto en fósforo extraíble $\left(55 \mathrm{mg} \mathrm{kg}^{-1}\right)$ y medio en bases intercambiables de potasio $\left(210 \mathrm{mg} \mathrm{kg}^{-1}\right)$, calcio (1928 $\mathrm{mg} \mathrm{kg}^{-1}$ ) y magnesio $\left(190 \mathrm{mg} \mathrm{kg}^{-1}\right)$. En el CEIXTA, el suelo es de textura migajón arenosa, con pH moderadamente ácido (5.43), bajo en su contenido de materia orgánica (1.01\%) y nitrógeno $\left(9 \mathrm{mg} \mathrm{kg}^{-1}\right)$, alto en fósforo $\left(51 \mathrm{mg} \mathrm{kg}^{-1}\right) \mathrm{y}$ medio en potasio $\left(175 \mathrm{mg} \mathrm{kg}^{-1}\right)$, calcio $\left(1053 \mathrm{mg} \mathrm{kg}^{-1}\right)$ y magnesio intercambiables (166 $\left.\mathrm{mg} \mathrm{kg}^{-1}\right)$. En el $\mathrm{CECECH}$, el suelo es migajón arcillo arenoso, con $\mathrm{pH}$ neutro (6.9), bajo en su contenido de materia orgánica $(1.1 \%)$, alto en nitrógeno inorgánico (106 $\left.\mathrm{mg} \mathrm{kg}^{-1}\right)$, calcio (3329 $\mathrm{mg} \mathrm{kg}^{-1}$ ) y magnesio (1158 $\mathrm{mg} \mathrm{kg}^{-1}$ ), muy alto en fósforo orgánico $\left(175 \mathrm{mg} \mathrm{kg}^{-1}\right)$ y medio en su contenido de potasio $\left(135 \mathrm{mg} \mathrm{kg}^{-1}\right)$.

\section{Diseño Experimental}

En cada sitio se condujo un ensayo uniforme de rendimiento, en el que se evaluaron 11 líneas seleccionadas durante el ciclo invierno-primavera
2013, en ensayos de riego-sequía, las cuales fueron: NGO-17-99, ELS-9-27, NGO-07022, Jamapa Plus, CIAT-103-25, SEQ-344-21, SCN-2, SEN-56, SEN-70, NCB-229 y X02-33-153, junto con las variedades Negro Tacaná, y Negro Jamapa (testigos regionales). Los ensayos se instalaron en el mes de octubre a una densidad de 250000 plantas ha-1, en los que hubo una emergencia mayor a $90 \%$ en los 13 genotipos de frijol, en los tres sitios experimentales. Se utilizó el diseño experimental bloques completos al azar con tres repeticiones y parcelas de tres surcos de $5 \mathrm{~m}$ de longitud, donde la parcela útil correspondió al surco central.

\section{Manejo Agronómico del Experimento}

Durante la fase vegetativa del cultivo se realizó fertilización mineral al suelo con la dosis 40-40-00 $\mathrm{kg} \mathrm{ha}^{-1}$ de $\mathrm{N}, \mathrm{P}_{2} \mathrm{O}_{5}$ y $\mathrm{K}_{2} \mathrm{O}$, respectivamente, utilizando como fuentes de fertilizante: urea y fosfato diamónico, grado 18-46-00, la cual se complementó con una fertilización foliar a base de N-P-K $\left(11.47 \% \mathrm{~N}, 8 \% \mathrm{P}_{2} \mathrm{O}_{5}, 6 \% \mathrm{~K}_{2} \mathrm{O}\right)$ y elementos menores $(0.036 \% \mathrm{~B}, 0.040 \% \mathrm{Cu}, 0.050 \%$ Fe, $0.005 \%$ Mo, $0.080 \%$ Zn y $0.230 \% \mathrm{~S}, 0.025 \%$ Ca y $0.002 \mathrm{Co}$ ) a razón de $2 \mathrm{~L} \mathrm{ha}^{-1}$ de producto comercial. En el CECECH y en el CEIXTA, la maleza de hoja ancha y angosta se controlaron manualmente con azadón, mientras que en La Candelaria, el control se realizó con los herbicidas fomesafén y fluazifopbutil, en dosis de $750+750 \mathrm{~mL} \mathrm{ha}^{-1}$ de producto comercial, cuyo control se complementó con una labor de cultivo realizado a los 27 días después de la siembra (Esquivel et al., 1997). La presencia de doradilla (Diabrotica balteata LeConte) en los ensayos del CECECH y La Candelaria se controló con dos aplicaciones de cipermetrina en dosis de $200 \mathrm{~mL} \mathrm{ha}^{-1}$ de producto comercial, en tanto que, en el CEIXTA la misma plaga y la chicharrita (Empoasca kraemeri Ross y Moore), se controlaron con dos aplicaciones de insecticida a base de imidacloprid + betacyfluthrin, en dosis de $200 \mathrm{~mL} \mathrm{ha}^{-1}$ de producto comercial y una de cipermetrina con $100 \mathrm{~mL} \mathrm{ha}^{-1} \mathrm{de}$ producto comercial.

\section{Variables Explicativas y de Respuesta}

Durante el ciclo del cultivo, se cuantificó la precipitación pluvial y la humedad del suelo. El registro de la precipitación pluvial en los sitios experimentales, se realizó con pluviómetros de PVC, haciendo las lecturas 
en probetas. La humedad del suelo se obtuvo con el método gravimétrico, en muestras tomadas una vez por semana, en los estratos de 0-15, 15-30 y $30-45 \mathrm{~cm}$, durante el ciclo del cultivo. Los datos de humedad del suelo se expresaron como humedad aprovechable y se realizó un balance de humedad para determinar el grado de sequía, en la cual se desarrollaron los genotipos de frijol, en los tres sitios experimentales.

Las variables de respuesta fueron: días a floración media de los genotipos, la cual se cuantificó a partir de la siembra hasta que $50 \%$ de las plantas tenían por lo menos una flor abierta; días a madurez fisiológica que se midió a partir de la siembra hasta que en $50 \%$ de las plantas de cada genotipo, sus vainas cambiaron de color verde a amarillo o morado, así como peso de 100 semillas en gramos y rendimiento de grano en kilogramos por hectárea ajustado al 14\% de humedad.

\section{Análisis Estadístico}

Se realizaron análisis de varianza de las variables de respuesta por localidad, así como un análisis conjunto (localidades-genotipos) de los tres ambientes de evaluación. Para la separación de promedio se aplicó la prueba basada en la Diferencia Mínima Significativa al 5\% de probabilidad de error (DMS, $\alpha=0.05)$ (SAS Institute, 1999).

\section{RESULTADOS Y DISCUSIÓN}

\section{Precipitación Pluvial}

En el CEIXTA se registraron lluvias frecuentes, con una precipitación pluvial acumulada hasta la madurez fisiológica del cultivo de $391.6 \mathrm{~mm}$ (Cuadro 1), por lo que prácticamente las plantas de frijol se desarrollaron sin estrés por humedad; en tanto que, en el CECECH y en La Candelaria, en ese periodo de desarrollo se tuvieron precipitaciones pluviales acumuladas de
97.7 y $52.1 \mathrm{~mm}$ (Cuadro 1), respectivamente, con restricciones de humedad durante la etapa reproductiva del cultivo (Fernández et al., 1985).

\section{Balance de Humedad del Suelo}

Los suelos en los cuales se estableció el ensayo presentaron una capacidad de campo (CC) de 23.6, 20.3 y $13 \%$ en los sitios La Candelaria, CEIXTA y CECECH, respectivamente, con un punto de marchitez permanente (PMP) de 12.5, 10.8 y 6\%, en los mismos sitios de prueba, lo que permitió una humedad aprovechable de $11.1,9.5$ y $7 \%$, de acuerdo con la metodología propuesta por Hillel (1980).

En el sitio de La Candelaria se observó menor variación de la humedad aprovechable entre las diferentes profundidades de suelo y fechas de muestreo, así como un descenso paulatino de ésta, desde la siembra hasta la madurez fisiológica del frijol, lo cual se asocia al comportamiento de la precipitación pluvial ocurrida en ese sitio de prueba. A su vez, en el $\mathrm{CECECH}$ se tuvo un comportamiento irregular, con alta variación de la humedad aprovechable del suelo entre las diferentes fechas de muestreo, sobre todo antes de la floración de los genotipos. Después de la floración hasta la etapa de madurez fisiológica se observó déficit hídrico en los tres estratos de suelo medidos, la cual fue más severa que en La Candelaria, por la baja capacidad de retención de humedad del suelo (Figura 1).

En el CEIXTA, el comportamiento de la humedad aprovechable durante todo el ciclo del cultivo fue muy variable dentro y entre fechas de muestreo, observándose mayor humedad en el estrato $0-15 \mathrm{~cm}$, con promedio de $87.5 \%$, que en los estratos $15-30 \mathrm{~cm}$ y $30-45 \mathrm{~cm}$, en donde se obtuvieron promedios de 58.4 y $53.4 \%$, respectivamente (Figura 1). En esta localidad se tuvieron mejores condiciones de humedad para el cultivo, que en los otros dos sitios de evaluación, cuya humedad aprovechable en la etapa de madurez

Cuadro 1. Precipitación pluvial ( $\mathrm{mm})$ ocurrida en los sitios experimentales en las diferentes etapas vegetativas del cultivo de frijol. Ciclo otoño-invierno de 2013-2014.

\begin{tabular}{|c|c|c|c|c|}
\hline Localidad/municipio/estado & Fecha de siembra & Fase vegetativa & Fase reproductiva & Total en el ciclo \\
\hline & & \multicolumn{3}{|c|}{$\ldots \ldots \ldots$. . Precipitación pluvial $(\mathrm{mm}) \ldots \ldots$} \\
\hline CECECH, Ocozocoautla, Chiapas & 1-Oct-13 & 97.7 & 0.0 & 97.7 \\
\hline La Candelaria, Medellín, Veracruz & 23-Oct-13 & 52.1 & 0.0 & 52.1 \\
\hline CEIXTA, Martínez de la Torre, Veracruz & 25 -Oct-13 & 256.0 & 135.6 & 391.6 \\
\hline
\end{tabular}



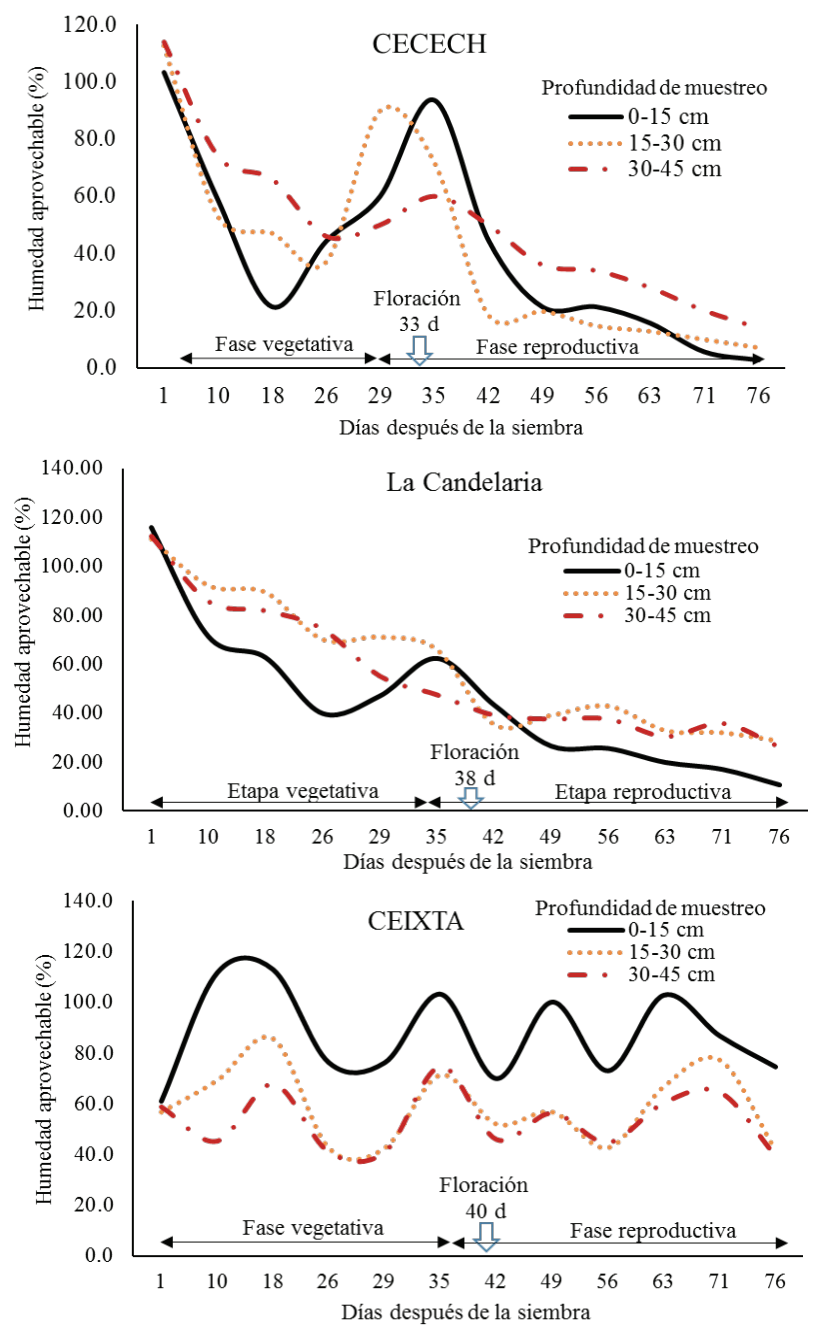

Figura 1. Humedad aprovechable del suelo en el Campo Experimental Centro de Chiapas (CECECH), La Candelaria y Campo Experimental Ixtacuaco (CEIXTA), durante el ciclo otoño-invierno 2013-2014.

fisiológica, en promedio de los tres estratos fue de $51.9 \%$, en tanto que en La Candelaria y en el CECECH la humedad aprovechable promedio en la misma etapa fue de 28.5 y $6.9 \%$, respectivamente.

La humedad aprovechable promedio de los tres estratos del suelo $(0-45 \mathrm{~cm})$ desde la siembra hasta la floración de la mayoría de los genotipos fue de 73.2, 67 y $61.9 \%$, equivalente a una humedad del suelo de 20.6, 17.2 y $10.3 \%$, en los sitios de La Candelaria, CEIXTA y CECECH, respectivamente. Después de este período, hacia la etapa terminal del cultivo, la humedad aprovechable promedio prevaleciente en los sitios respectivos fue de 34.3, 65.9 y $15.5 \%$, equivalente a una humedad del suelo de 16.3, $17.1 \mathrm{y}$ $7.1 \%$. Este comportamiento pone de manifiesto que en el CEIXTA no hubo diferencia en la humedad del suelo antes y después de floración; mientras que, en La Candelaria y en el CECECH, las diferencias en humedad aprovechable antes y después de floración fueron de 38.9 y $46.4 \%$, respectivamente.

\section{Floración Media, Madurez Fisiológica, Peso de 100 Semillas y Rendimiento de Grano}

En las tres localidades se detectó significancia estadística entre tratamientos $(P \leq 0.01)$ en las variables días a floración media, madurez fisiológica y peso de 100 semillas. Respecto al rendimiento de grano, sólo en las dos localidades de Veracruz se encontró efecto significativo entre tratamientos $(P \leq 0.01)$ (Cuadro 2).

En las tres localidades de prueba, las líneas SEN-56, NCB-229, SCN-2 y SEN-70, tuvieron un tiempo de floración significativamente más temprano que el resto de los genotipos. De acuerdo al análisis combinado, los días a floración media variaron significativamente entre ambientes de evaluación y genotipos. En la localidad de CEIXTA, se tuvo el mayor número de días a la floración media, los cuales fueron significativamente superiores, a los obtenidos en las otras dos localidades.

En el factor genotipo, las variedades Negro Tacaná y Negro Jamapa, junto con siete líneas, conformaron el grupo con el mayor número de días a la floración media, los cuales fueron estadísticamente superiores al de las cuatro líneas arriba indicadas, que en promedio presentaron su floración antes de los 35 días después de la siembra (Cuadro 3).

En los sitios de La Candelaria y CECECH en los que hubo ocurrencia de sequía, las líneas SCN-2, SEN-56 y SEN-70 fueron los genotipos más precoces, los cuales alcanzaron su madurez fisiológica entre los 63 y 67 días después de la siembra, también se observó menor tiempo a la madurez fisiológica en las líneas NCB-229, NGO 07022 y X02-33-153 (Cuadro 4). A su vez, en el CEIXTA, sólo las líneas SEN-56 y SEN-70, mostraron un tiempo significativamente menor a la madurez fisiológica que el resto de los genotipos (Cuadro 4).

En el análisis combinado se detectó significancia en la variable madurez fisiológica en los factores ambientes y genotipo $(P \leq 0.01)$. En la localidad del CEIXTA, la madurez fisiológica ocurrió en un tiempo significativamente más tardío, que en los otros dos sitios de evaluación (Cuadro 4). En el CECECH y en La Candelaria, la madurez ocurrió antes de los 70 días. En el factor genotipo, en promedio de 
Cuadro 2. Cuadrados medios y significancia estadística de días a floración media, días a madurez fisiológica, peso de 100 semillas y rendimiento de grano de genotipos de frijol negro evaluados en dos localidades de Veracruz y una en Chiapas. Ciclo otoño-invierno 2013-2014.

\begin{tabular}{|c|c|c|c|c|}
\hline FV & GL & La Candelaria, Veracruz & CEIXTA, Veracruz & CECECH, Chiapas \\
\hline \multicolumn{5}{|c|}{ Días a floración media } \\
\hline Tratamientos & 12 & $32.363281 * *$ & $13.324219 * *$ & $8.564453 * *$ \\
\hline Bloques & 2 & 0.027344 & 0.691406 & 3.873047 \\
\hline Error & 24 & 0.414388 & 1.414876 & 2.288249 \\
\hline Total & 38 & & & \\
\hline CV $(\%)$ & & 1.70 & 3.00 & 4.62 \\
\hline \multicolumn{5}{|c|}{ Días a madurez fisiológica } \\
\hline Tratamientos & 12 & $26.195313 * *$ & $4.076823 * *$ & $15.635417 * *$ \\
\hline Bloques & 2 & 0.101563 & 2.484375 & 4.023438 \\
\hline Error & 24 & 3.242188 & 0.904297 & 2.054036 \\
\hline Total & 38 & & & \\
\hline CV $(\%)$ & & 2.6 & 1.18 & 2.12 \\
\hline \multicolumn{5}{|c|}{ Peso de 100 semillas } \\
\hline Tratamientos & 12 & $29.8804 * *$ & $43.2799 * *$ & $7.9188 * *$ \\
\hline Bloques & 2 & 0.0254 & 3.3193 & 7.8721 \\
\hline Error & 24 & 0.6367 & 2.5074 & 1.7329 \\
\hline Total & 38 & & & \\
\hline $\mathrm{CV}(\%)$ & & 3.51 & 6.54 & 7.65 \\
\hline \multicolumn{5}{|c|}{ Rendimiento de grano } \\
\hline Tratamientos & 12 & $82329.33 * *$ & $97751.33 * *$ & $11772.08 \mathrm{~ns}$ \\
\hline Bloques & 2 & 5542.00 & 13418.00 & 6462.00 \\
\hline Error & 24 & 17784.83 & 27835.66 & 7389.96 \\
\hline Total & 38 & & & \\
\hline CV $(\%)$ & & 13.38 & 13.86 & 15.01 \\
\hline
\end{tabular}

los tres ambientes de evaluación, las líneas SEN-70, SEN-56 y SCN-2, presentaron un número de días a la madurez significativamente inferior, a los del resto de los genotipos, incluidas las variedades Negro Tacaná y Negro Jamapa (Cuadro 4).

La línea SCN-2 fue el genotipo que se ubicó en el grupo con mayor peso de 100 semillas, tanto en las localidades en que hubo ocurrencia de sequía, como sin estrés de humedad, es decir, en los tres ambientes de evaluación, con un valor estadísticamente superior al de las variedades comerciales Negro Tacaná y Negro Jamapa (Cuadro 5). Esta característica también varió significativamente entre ambientes y genotipos $(P \leq 0.01)$. En el factor genotipo, la línea SCN-2 obtuvo en promedio un peso mayor a $27 \mathrm{~g}$ por 100 semillas, significativamente superior a los obtenidos por el resto de los genotipos, los cuales registraron pesos menores a $25 \mathrm{~g}$; en el factor ambiente en promedio el mayor peso se obtuvo en el CEIXTA, con diferencia significativa, respecto a los sitios con presencia de sequía terminal (Cuadro 5).

La presencia de estrés hídrico se asoció con menor rendimiento de grano, encontrándose que ambos sitios con sequía presentaron los menores promedios de rendimiento de grano, con diferencia significativa respecto a la localidad CEIXTA (Cuadro 6). Siete líneas sobresalieron por su alto rendimiento promedio, el cual fue significativamente superior al del resto de los genotipos, incluidas las variedades Negro Tacaná y Negro Jamapa (testigos regionales); de este grupo destaca la línea SEN-70, la cual mostró la mayor producción, tanto en los ambientes en que hubo 
Cuadro 3. Días a floración media de genotipos de frijol negro evaluados en dos localidades de Veracruz y uno en Chiapas. Ciclo otoñoinvierno 2013-2014.

\begin{tabular}{|c|c|c|c|c|}
\hline Genotipo & La Candelaria, Veracruz & CEIXTA, Veracruz & CECECH, Chiapas & Promedio \\
\hline NGO 17-99 & $40.3 *$ & $40.3 *$ & $32.7 *$ & $37.8 *$ \\
\hline ELS 9-27 & $40.0 *$ & $41.3 *$ & 31.7 & $37.7 *$ \\
\hline Jamapa Plus & $40.0 *$ & $41.0 *$ & $34.0 *$ & $38.3^{*}$ \\
\hline NGO 07022 & $40.0 *$ & $40.3 *$ & $33.7 *$ & $38.0^{*}$ \\
\hline CIAT-103-25 & $40.0 *$ & $39.7 *$ & $34.0 *$ & $37.9 *$ \\
\hline SEQ-344-21 & $40.0 *$ & $41.7 *$ & $35.0 *$ & $38.9 *$ \\
\hline SCN-2 & 33.0 & 37.7 & 31.3 & 34.0 \\
\hline SEN-56 & 33.0 & 34.7 & 29.3 & 32.3 \\
\hline SEN-70 & 33.0 & 39.0 & 31.7 & 34.6 \\
\hline NCB-229 & 33.7 & 36.7 & 31.3 & 33.9 \\
\hline $\mathrm{X} 02-33-153$ & 39.0 & $40.7 *$ & 32.0 & $37.2 *$ \\
\hline N. Tacaná & $40.3 *$ & $41.7 *$ & $34.0 *$ & $38.7 *$ \\
\hline N. Jamapa & $40.0 *$ & $40.3 *$ & $35.0 *$ & $38.4^{*}$ \\
\hline Promedio & 37.9 & $39.6 *$ & 32.7 & 36.7 \\
\hline DMS (0.05) & 1.085 & 2.005 & 2.549 & 2.202 \\
\hline
\end{tabular}

* Genotipos estadísticamente superiores, según la Diferencia Mínima Significativa (0.05).

Cuadro 4. Días a madurez fisiológica de genotipos de frijol negro evaluados en dos ambientes de Veracruz y uno en Chiapas. Ciclo otoño-invierno 2013-2014.

\begin{tabular}{lcccc}
\hline Genotipo & La Candelaria, Veracruz & CEIXTA, Veracruz & CECECH, Chiapas & Promedio \\
\hline NGO 17-99 & $71.7 *$ & $81.3 *$ & $70.3 *$ & $74.4^{*}$ \\
ELS 9-27 & $68.7 *$ & $82.0 *$ & $69.0 *$ & $73.2 *$ \\
Jamapa Plus & $70.0 *$ & $81.7 *$ & $69.3 *$ & $73.7 *$ \\
NGO 07022 & $71.7 *$ & $81.0 *$ & 66.0 & $72.9 *$ \\
CIAT-103-25 & $69.7 *$ & $81.0 *$ & $69.3 *$ & $73.3 *$ \\
SEQ-344-21 & $70.0 *$ & $81.3 *$ & $69.7 *$ & $73.7 *$ \\
SCN-2 & 67.0 & $80.7 *$ & 64.0 & 70.6 \\
SEN-56 & 63.7 & 78.0 & 65.0 & 68.9 \\
SEN-70 & 63.0 & 79.0 & 66.0 & 69.3 \\
NCB-229 & $71.7 *$ & $80.7 *$ & 64.7 & $72.3 *$ \\
X02-33-153 & $69.7 *$ & $81.3 *$ & 66.7 & $72.6 *$ \\
N. Tacaná & $71.7 *$ & $81.7 *$ & $69.7 *$ & $74.3 *$ \\
N. Jamapa & $71.3 *$ & $82.0 *$ & $69.7 *$ & $74.3 *$ \\
Promedio & 69.2 & $80.9 *$ & 67.6 & 2.415 \\
DMS (0.05) & 3.034 & 1.603 & 72.6 \\
\hline
\end{tabular}

* Genotipos estadísticamente superiores, según la Diferencia Mínima Significativa (0.05).

ocurrencia de sequía terminal, como en la localidad de CEIXTA, en donde no hubo sequía (Cuadro 6).

De acuerdo a los resultados de balance de humedad aprovechable de suelo, en dos de los tres ambientes de evaluación, hubo sequía, por lo que puede aseverarse que los genotipos de frijol evaluados en La Candelaria $\mathrm{y}$ en el CECECH, sufrieron estrés hídrico (Allen et al., 2006), condiciones favorables, para identificar genotipos con mejor respuesta en rendimiento de grano bajo condiciones de déficit de humedad, mientras 
Cuadro 5. Peso de 100 semillas (g) de genotipos de frijol negro evaluados en dos ambientes de Veracruz y uno en Chiapas. Ciclo otoñoinvierno de 2013-14.

\begin{tabular}{|c|c|c|c|c|}
\hline Genotipo & La Candelaria, Veracruz & CEIXTA, Veracruz & CECECH, Chiapas & Promedio \\
\hline NGO 17-99 & 23.3 & 24.0 & 17.0 & 21.5 \\
\hline ELS 9-27 & 19.7 & 20.4 & 17.3 & 19.1 \\
\hline Jamapa Plus & 19.7 & 22.1 & 15.7 & 19.1 \\
\hline NGO 07022 & 21.7 & 22.1 & 17.0 & 20.3 \\
\hline CIAT-103-25 & 22.0 & 23.7 & 16.3 & 20.7 \\
\hline SEQ-344-21 & 20.3 & 19.8 & 16.0 & 18.7 \\
\hline SCN-2 & $29.7 *$ & $33.2 *$ & $20.3 *$ & $27.7 *$ \\
\hline SEN-56 & 26.0 & 27.0 & $19.3 *$ & 24.1 \\
\hline SEN-70 & 24.0 & 27.4 & $19.7 *$ & 23.7 \\
\hline NCB-229 & 27.0 & 28.3 & 17.7 & 24.3 \\
\hline X02-33-153 & 20.3 & 22.5 & 15.3 & 19.4 \\
\hline N. Tacaná & 21.7 & 21.0 & 16.0 & 19.6 \\
\hline N. Jamapa & 20.0 & 23.2 & 16.0 & 19.7 \\
\hline Promedio & 22.7 & $24.2 *$ & 17.2 & 21.4 \\
\hline DMS (0.05) & 1.345 & 2.669 & 2.218 & 2.477 \\
\hline
\end{tabular}

* Genotipos estadísticamente superiores, según la Diferencia Mínima Significativa (0.05).

Cuadro 6. Rendimiento de grano $\left(\mathrm{kg} \mathrm{ha}^{-1}\right)$ de genotipos de frijol negro evaluados en dos ambientes de Veracruz y uno en Chiapas. Ciclo otoño-invierno 2013-2014.

\begin{tabular}{lcccc}
\hline Genotipo & La Candelaria, Veracruz & CEIXTA, Veracruz & CECECH, Chiapas & Promedio \\
\hline NGO 17-99 & $1141.3 *$ & $1279.3 *$ & 622.7 & $1014.4 *$ \\
ELS 9-27 & 719.7 & 998.0 & 510.7 & 742.8 \\
Jamapa Plus & $1218.3 *$ & $1263.0 *$ & 530.7 & 624.0 \\
NGO 07022 & $1034.0 *$ & $1329.3 *$ & 501.3 & $995.8 *$ \\
CIAT-103-25 & 998.0 & 840.0 & 613.3 & 779.8 \\
SEQ-344-21 & 1009.3 & 885.7 & 625.3 & 836.1 \\
SCN-2 & $1043.7 *$ & $1271.3 *$ & 606.7 & $980.1 *$ \\
SEN-56 & $1057.3 *$ & $1262.0 *$ & 686.7 & $975.3 *$ \\
SEN-70 & $1253.3 *$ & $1427.3 *$ & 568.0 & $1122.4 *$ \\
NCB-229 & 840.7 & $1241.3 *$ & 538.7 & 883.3 \\
X02-33-153 & $1044.3 *$ & $1337.0 *$ & 468.0 & $973.3 *$ \\
N. Tacaná & 747.0 & $1199.7 *$ & 549.3 & 804.9 \\
N. Jamapa & 854.3 & $1318.0 *$ & 572.7 & ns \\
Promedio & 997.0 & $1204.0 *$ & 281.167 & 924.6 \\
DMS (0.05) & 224.744 & & 198.502 \\
* Genotipos estadísticamente superiores, según la Diferencia Mínima Significativa $(0.05)$. &
\end{tabular}

que, en el CEIXTA, las condiciones de humedad que prevalecieron durante el ciclo del cultivo, no permitieron el estrés hídrico de los genotipos de frijol.

La significancia estadística entre tratamientos para días a floración media, a madurez fisiológica y peso de 100 semillas, se debe principalmente a la diferencia genética y a su respuesta a los diferentes ambientes de prueba. Respecto a la significancia en el rendimiento 
de grano, se infiere que en las dos localidades con diferencia significativa, los genotipos mostraron diferente potencial productivo, bajo las condiciones de humedad residual en que fueron evaluados.

Generalmente, la precocidad en la floración se asocia a un adelanto en los días a madurez fisiológica de los genotipos (Acosta-Díaz et al., 2004; Manjeru et al., 2007), premisa que se cumplió en forma parcial en este estudio. La mayoría de los genotipos que mostraron precocidad a la floración, también lo hicieron a la madurez fisiológica, tanto en las localidades en que hubo ocurrencia de sequía, como en el CEIXTA en donde no hubo estrés por humedad. En esta última localidad, las líneas mostraron menor precocidad y sólo SEN-70 y SEN-56 alcanzaron su madurez en un tiempo significativamente menor. De acuerdo con AcostaDíaz et al. (2011), el criterio de la precocidad es una característica de escape importante para la selección de genotipos tolerantes a sequía.

La madurez fisiológica más tardía en la localidad CEIXTA, se debió principalmente, a que se tuvieron mejores condiciones de humedad durante todo el ciclo del cultivo, que favorecieron un mayor tiempo a la madurez fisiológica (Ramírez-Vallejo y Kelly, 1998). A su vez, en el CECECH y en La Candelaria, el menor tiempo a madurez fue debido a las condiciones de deficiencia de humedad presentadas, principalmente después de la etapa de floración del cultivo, lo que provocó adelanto en la madurez fisiológica, que de acuerdo con Rosales-Serna et al. (2001), el aceleramiento de la madurez ocurre cuando la sequía se prolonga durante la fase reproductiva y no se presentan condiciones favorables para la recuperación.

La precocidad de las líneas SEN-70, SEN-56 y SCN-2 se ha documentado en otros estudios de evaluación realizados en el sureste de México (TosquyValle et al., 2014), la cual es muy deseable, debido a que las plantas de frijol pueden escapar a la falta de humedad (Acosta-Díaz et al., 2004), ocasionada por la ocurrencia de sequía terminal, que reduce el rendimiento de frijol, en las siembras comerciales de humedad residual de los estados de Veracruz y Chiapas (López-Salinas et al., 2008).

El mayor peso de 100 semillas de la línea $\mathrm{SCN}-2$, en los tres ambientes de evaluación, obedece principalmente, a que este material es de grano de mayor tamaño, que el tipo tropical local, característica que está relacionada con un mayor peso de semilla
(Tosquy-Valle et al., 2014). Entre ambientes, el mayor peso de 100 semillas observado en el CEIXTA fue debido principalmente a la mayor humedad que prevaleció durante el ciclo de desarrollo del cultivo, la cual favoreció una mayor capacidad de las plantas de frijol de translocar asimilados hacia el grano (Rao, 2001). En el factor genotipo, el peso promedio de 100 semillas mayor a $27 \mathrm{~g}$ de SCN-2, indica que el grano de esta línea es considerado como de tamaño medio y en el sureste de México, la mayor demanda comercial de frijol negro, es de grano de tamaño pequeño, con un peso menor a $25 \mathrm{~g}$ por 100 semillas (Rosales-Serna et al., 2003; López-Salinas et al., 2012; SNICS, 2013).

El mayor promedio en rendimiento de grano obtenido en la localidad de CEIXTA, se debió principalmente a una mayor y mejor distribución de la lluvia durante el ciclo del cultivo, principalmente de las etapas de floración al llenado de vainas, en donde el cultivo recibió más de $90 \mathrm{~mm}$ de precipitación pluvial, suficientes para obtener buen llenado del grano (Ruíz et al., 1999; Martínez et al., 2008) y por consiguiente una mayor humedad aprovechable del suelo para las plantas de frijol (Cuadro 1 y Figura 1); por el contrario, el menor rendimiento promedio observado en el $\mathrm{CECECH}$, fue debido principalmente a dos factores: 1) A que el suelo tuvo menor capacidad de retención de humedad, lo cual implicó una humedad aprovechable baja para el cultivo (Figura 1) y 2) A la ocurrencia de sequía durante la etapa reproductiva del cultivo, lo que afectó adversamente el rendimiento de grano (Ruíz et al., 1999; Acosta-Díaz et al., 2009). Aunque en la Candelaria se tuvo menor precipitación pluvial que en el CECECH, el rendimiento fue mayor, debido principalmente a una mayor capacidad de retención de humedad en el suelo (Figura 1).

En el factor genotipo se identificó un grupo de líneas con mayor potencial de rendimiento que las variedades comerciales Negro Tacaná y Negro Jamapa, bajo condiciones de humedad residual en los estados de Veracruz y Chiapas. La mayor producción mostrada en la línea SEN-70, principalmente obedece, a sus características genéticas intrínsecas, de precocidad, alto rendimiento y tolerancia al estrés por humedad (Tosquy-Valle et al., 2014). Cabe destacar, que el grano de esta línea, reúne las características de tipo de frijol negro, opaco y de tamaño pequeño, que demandan los consumidores de esta región (Rosales-Serna et al., 2003; López-Salinas et al., 2012). 


\section{CONCLUSIONES}

SEN-70, NGO-17-99 y Jamapa Plus, junto con otras cuatro líneas, mostraron una producción promedio significativamente mayor que el de las variedades comerciales Negro Tacaná y Negro Jamapa, bajo condiciones de humedad residual. La línea SEN-70 fue la de mayor rendimiento con y sin estrés por humedad y su precocidad le permitió escapar a la sequía terminal.

\section{LITERATURA CITADA}

Acosta, J. A., E. Acosta, S. Padilla-Ramírez, Ma. A. Goytia, R. Rosales y E. López. 1999. Mejoramiento de la resistencia a la sequía del frijol común en México. Agron. Mesoam. 10: 83-90.

Acosta-Díaz, E., C. Trejo-López, L. M. Ruíz-Posadas, J. S. PadillaRamírez y J. A. Acosta.-Gallegos. 2004. Adaptación del frijol a sequía en la etapa reproductiva. Terra Latinoamericana 22: 49-58.

Acosta-Díaz, E., I. Hernández-Torres, R. Rodríguez-Guerra, J. A. Acosta-Gallegos, J. Pedroza-Flores, M. D. Amador-Ramírez y J. S. Padilla-Ramírez. 2011. Efecto de la sequía en la producción de biomasa y grano de frijol. Rev. Mex. Cienc. Agríc. 2: 249-263.

Acosta-Díaz, E., J. A. Acosta-Gallegos, C. Trejo-López, J. S. PadillaRamírez, and M. D. Amador-Ramírez. 2009. Adaptation traits in dry bean cultivars grown under drought stress. Agric. Téc. Méx. 35: 416-425.

Allen, R. G., L. S. Pereira, D. Raes y M. Smith. 2006. Evapotranspiración del cultivo. Guías para la determinación de los requerimientos de agua de los cultivos. Estudio FAO. Riego y Drenaje No. 56. Organización de las Naciones Unidas para la Agricultura y la Alimentación. Roma, Italia. ISBN 92-5-304219-2.

Díaz-Padilla, G., J. A. Ruíz-Corral, M. A. Cano-García, V. Serrano-Altamirano y G. Medina-García. 2006. Estadísticas climatológicas básicas del estado de Veracruz (período 1961-2003). Libro Técnico Núm. 13. SAGARPA. INIFAP. CIRGOC. Campo Experimental Cotaxtla. Veracruz, México. ISBN: 970-43-0020-4.

Norma Oficial Mexicana NOM-021-RECNAT-2000. 2000. Que establece las especificaciones de fertilidad, salinidad y clasificación de suelos. Estudios, muestreo y análisis. Diario Oficial de la Federación, 17 de octubre de 2000. México, D. F.

Emam, Y., A. Shekoofa, F. Salehi, and A. H. Jalali. 2010. Water stress effects on two common bean cultivars with contrasting growth habits. American-Eurasian J. Agric. Environ. Sci. 9: 495-499.

Esquivel, V. A., O. Cano y E. López. 1997. Control químico de malezas en frijol (Phaseolus vulgaris L.) en el estado de Veracruz. Agron. Mesoam. 8: 53-58.

Fernández, F., P. Geps y M. López. 1985. Etapas de desarrollo en la planta de frijol. pp. 61-78. In: M. López, F. Fernández y A. van Schoonhoven. (eds.). Frijol: Investigación y producción : Referencia de los cursos de capacitación sobre frijol dictados por el Centro Internacional de Agricultura Tropical. PNUDCIAT. Cali, Colombia.
Frahm, M., J. C. Rosas, N. Mayek-Pérez, E. López-Salinas, J. A. Acosta-Gallegos y J. D. Kelly. 2003. Resistencia a sequía terminal en frijol negro tropical. Agron. Mesoam. 14: 143-150.

García, E. 2004. Modificaciones al sistema de clasificación climática de Köppen. Instituto de Geografía, UNAM. México, D. F.

Hillel, D. 1980. Applications of soil physics. Academic Press. New York, NY, USA.

López-Salinas E., O. H. Tosquy-Valle, F. J. Ugalde-Acosta y J. A. Acosta-Gallegos. 2008. Rendimiento y tolerancia a sequía de genotipos de frijol negro en el estado de Veracruz. Rev. Fitotec. Mex. 31: 35-39.

López-Salinas, E., O. H. Tosquy-Valle, J. A. Acosta-Gallegos, B. Villar-Sánchez, and F. J. Ugalde-Acosta. 2011. Drought resistance of tropical dry black bean lines and cultivars. Trop. Subtrop. Agroecosyst. 14: 749-755.

López-Salinas, E., O. H. Tosquy-Valle, Y. Jiménez-Hernández, R. A. Salinas-Pérez, B. Villar-Sánchez y J. A. Acosta-Gallegos. 2012. Rendimiento y adaptación de la variedad de frijol Negro Comapa en dos regiones de México. Rev. Fitotec. Mex. 35: 309-315.

Manjeru, P., T. Madanzi, B. Makeredza, A. Nciizah, and M. Sithole. 2007. Effect of water stress at different growth stages on components and grain yield of common bean (Phaseolus vulgaris L.). African Crop Sci. Conf. Proc. 8: 299-303.

Martínez-Gamiño, M. A., E. S. Osuna-Ceja, J. S. Padilla-Ramírez, J. A. Acosta-Gallegos y C. Loredo-Osti.2008. Tecnología para la producción de frijol en el norte centro de México. Libro Técnico No. 4. Instituto Nacional de Investigaciones Forestales Agrícolas y Pecuarias. Centro de Investigación Regional del Noreste. Campo Experimental San Luis. San Luis Potosí, México. ISBN: 978-607-425-030-5.

Molina, J. C., V. Moda-Cirino, N. da Silva Fonseca-Júnior, R. Teixeira de Faria, and D. Destro. 2001. Response of common bean cultivars and lines to water stress. Crop Breed. Appl. Biotechnol. 1: 363-372.

Ramírez-Vallejo, P. and J. D. Kelly.1998. Traits related to drought resistance in common bean. Euphitica 99: 127-136.

Rao, I. M. 2001. Role of physiology in improving crop adaptation to abiotic stresses in the tropics: The case of common bean and tropical forages. pp. 583-613. In: M. Pessarakli. (ed.). Handbook of plant and crop physiology. Marcel Dekker Inc. New York, NY, USA.

Rosales-Serna, R., J. A. Acosta-Gallegos, R. P. Durán-Durán, H. Guillén-Andrade, P. Pérez-Herrera, G. Esquivel-Esquivel y J. S. Muruaga-Martínez. 2003. Diversidad genética del germoplasma mejorado de frijol (Phaseolus vulgaris L.) en México. Agric. Téc. Méx. 29: 11-24.

Rosales-Serna, R., R. Ochoa-Márquez y J. A. Acosta-Gallegos. 2001. Fenología y rendimiento del frijol en el altiplano de México y su respuesta al fotoperiodo. Agrociencia 35: 513-523.

Ruíz-Corral, J. A., G. Medina G., I. J. González A., C. Ortiz T., H. E. Flores L., R. A. Martínez P. y K. F. Byerly M. 1999. Requerimientos agroecológicos de los cultivos. Libro Técnico Núm. 3. INIFAP-CIRPAC. Conexión Gráfica. Guadalajara, Jal., México.

SAS Institute. 1999. SAS/STAT user's guide. Versión 8.0. SAS Institute. Cary, NC, USA. 
Serrano-Altamirano, V., G. Díaz-Padilla, A. López-Luna, M. A. Cano-García, A. D. Báez-González y E. R. Garrido-Ramírez. 2006. Estadísticas climatológicas básicas del estado de Chiapas (período 1961-2003). Libro técnico Núm. 1. INIFAP, SAGARPA. CIRPAS. Ocozocoautla, Chis., México.

SIAP (Servicio de Información Agroalimentaria y Pesquera). 2014. Producción agropecuaria. Producción anual. Cierre de la producción agrícola por estado. Anuario estadístico de la producción agrícola. SAGARPA. http://www.siap.sagarpa. gob.mx. (Consulta: junio 4, 2014).
SNICS (Servicio Nacional de Inspección y Certificación de Semillas). 2013. Guía técnica para la descripción varietal. Frijol (Phaseolus vulgaris L.). SAGARPA, SNICS. Tlalnepantla, Edo. de Méx., México.

Tosquy-Valle, O. H., E. López-Salinas, N. Francisco-Nicolás, J. A. Acosta-Gallegos y B. Villar-Sánchez. 2014. Genotipos de frijol negro opaco resistentes a sequía terminal. Rev. Mex. Cienc. Agríc. 5: 1205-1217. 